\title{
291
}

\section{CONFIDENCE LIMITS FOR A CROSS-PRODUCT RATIO ${ }^{1}$}

\author{
SIR Ronald A. Fisher, w.R.s. \\ Division of Mathematical Statisties, C.S.I.R.O. \\ 1 Received for publication March 9, 1962.
}

If the observations in a $2 \times 2$ table are distinctly out of proportion (and indeed in other cases also) we may wish to set limits to the true cross-product ratıo, e.g. the observed table

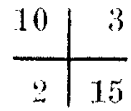

wives a crude ratio of 25 . How small could the true ratio be in reasonable consistency with the data?

If the expectations in the four classes were

the true ratio would be

$$
\begin{array}{l|r}
10-x & 3+x \\
\hline 2+x & 15-x
\end{array}
$$

and $x^{2}$ for the observations would be

so, if $x$ were $3 \cdot 0$

$$
x^{2}=x^{2}\left(\frac{1}{10-x}+\frac{1}{3+x}+\frac{1}{2+x}+\frac{1}{10-x}\right):
$$

with one degree of freedom.

$$
x^{2}=3^{2}(0 \cdot 59286)=5 \cdot 3357
$$

The exact probability of such a small sample of 30 giving 10 or more in the first quadrant is the partial sum of a hypergeometric series, and not easy to calculate, for if $\xi$ stand for the theoretical product ratio, the frequencies of 0 to 12 in the quadrant will be proportional to the terms

$$
1, \frac{13 \times 12}{1 \times 6}, \frac{13 \times 12 \times 12 \times 11}{1 \times 2 \times 6 \times 7} \xi^{2}, \ldots \frac{13 ! 12 ! 5 !}{(13-r) !(12-r) !(5+r) ! r !} \xi^{r} \ldots
$$

It would not be too difficult, as in the exact test for disproportionality, to calculate the last three terms for any chosen value of $\xi$, but for the ratio of these to the whole we would require the sum of the entire series, or

$$
F(-13,-12,6, \xi)
$$

which would be best obtained by calculating all the terms and summing them, a process too lengthy to be recommended.

Using Yates' adjustment, however, we can at once find

$$
\chi_{c}^{2}=(2 \cdot 5)^{2}(0 \cdot 59286)=3 \cdot 7054
$$

Further, taking $x=3 \cdot 1$ we have

$$
\chi_{c}^{2}=(2 \cdot 6)^{2}(0.58897)=3.9815 \text {. }
$$

Interpolating for the tabular entry $3 \cdot 841$, it appears that,

$$
x=3.0491
$$

and the cross-product ratio 2.720 .

So that it may be inferred from the data that the true crossproduct ratio exceeds 2.720 , unless a coincidence of one in forty has occurred. Similar limits can be set in both directions and at all levels of probability. 